\title{
Introduction : éduquer le regard rhétorique
}

\section{Victor Ferry et Benoît Sans}

\section{Q OpenEdition}

\section{Journals}

\section{Édition électronique}

URL : http://journals.openedition.org/rhetorique/402

DOI : 10.4000/rhetorique.402

ISSN : 2270-6909

\section{Éditeur}

UGA Éditions/Université Grenoble Alpes

\section{Édition imprimée}

ISBN : 978-284310-303-2

\section{Référence électronique}

Victor Ferry et Benoît Sans, «Introduction : éduquer le regard rhétorique », Exercices de rhétorique [En ligne], 5 | 2015, mis en ligne le 24 septembre 2015, consulté le 12 septembre 2020. URL : http:// journals.openedition.org/rhetorique/402 ; DOI : https://doi.org/10.4000/rhetorique.402

Ce document a été généré automatiquement le 12 septembre 2020

\section{(c) (i) (2)}

Les contenus de la revue Exercices de rhétorique sont mis à disposition selon les termes de la Licence Creative Commons Attribution - Pas d'Utilisation Commerciale - Partage dans les Mêmes Conditions 4.0 International. 


\title{
Introduction : éduquer le regard rhétorique
}

\author{
Victor Ferry et Benoît Sans
}

1 Partons d'une proposition radicale, au risque d'être imprudents: nous vivons en démocratie mais nous ne sommes pas démocrates. Nous bénéficions d'une liberté d'expression relativement bien garantie, nous avons un droit de regard sur les grandes questions ayant trait à la gestion de nos cités et, de temps à autre, nous avons même l'opportunité de porter au pouvoir ceux des représentants qui nous déplaisent le moins et de sanctionner ceux qui nous ont le plus déplu. Tout ceci ne fait pas de nous des démocrates. Imaginons, pour l'exercice, que notre système change radicalement. Imaginons, par exemple, qu'une idée toute simple gagne du terrain: il n'existe pas d'être humain tellement meilleur qu'un autre. Partant, il deviendrait saugrenu de donner autant de pouvoir à une seule personne, de laisser à un individu, aussi méritant soit-il, le soin d'assumer le destin de centaines, de milliers, de millions d'individus. Alors, nos représentants, que nous aimons tant haïr, nous apparaitront pour ce qu'ils sont : de petits hommes avec des responsabilités beaucoup trop grandes pour eux. Si cette idée gagne du terrain, nous pourrions avoir envie d'expérimenter une forme plus directe de démocratie et, à l'instar de nos lointains ancêtres, de nous emparer de la chose publique. Mais alors, c'est toute une gamme de compétences qu'il nous faudra (re)apprendre à apprivoiser. Citons-en quelques-unes : être capable de construire un discours cohérent, calibré et de le délivrer distinctement et sans appréhension face à un vaste auditoire qui n'a, a priori, aucune raison d'être bien disposé à notre égard ; être capable d'engager, de maintenir, de restaurer un terrain d'entente avec des personnes qui pour des raisons culturelles, sociales ou ethniques se situent en dehors de notre cercle empathique ; être capable de supporter les émotions du désaccord, de garder les idées claires tout en perdant la face, d'accepter la contradiction sans y voir une fatalité ; être capable, au plus profond d'un dialogue de sourds ${ }^{1}$ qui s'envenime, de prendre le recul théorique nécessaire pour voir en l'autre non pas un ennemi, qu'il faudrait supprimer, mais un autre orateur, que l'on pourra au moins respecter, pour la beauté du geste. C'est à la description de ces compétences rhétoriques à usage civique qu'est consacré ce dossier. Les articles qui le composent suivent une même 
méthodologie: rendre explicite du savoir pratique, révéler les gestes cognitifs et discursifs à l'œuvre derrière différentes techniques rhétoriques. Ces descriptions ont vocation à générer des prises de conscience, étape nécessaire à la maitrise de compétences. Avant d'y venir, nous souhaitons, en bons sophistes, relever un défi.

Dans le paragraphe qui précède, nous avons, dans la plus pure tradition rhétorique, commencé par l'éloge de notre art. La rhétorique est l'organon du politique, plus de démocratie demande plus de rhétorique et plus de rhétorique amène plus de démocratie. Mais a-t-on tant besoin de rhétorique? Les sciences cognitives nous ont enseigné que l'argumentation est un phénomène spontané: l'argumentation se déclenche automatiquement à la suite d'un conflit cognitif ${ }^{2}$. Les humains argumentent comme ils respirent, or, a-t-on besoin d'un professeur pour apprendre à respirer ? Cela est indéniable: spontanément, nous savons argumenter. Mais savons-nous bien argumenter? Sur cette question, la logique, devenue informelle puis pragmadialectique, dispose d'un certain pouvoir de conviction. Pour bien argumenter, il faut d'abord bien raisonner, ce qui suppose de renoncer à nos biais cognitifs et d'apprendre à rester sur les rails de l'argumentation vertueuse. De leur côté, les rhétoriciens se sont surtout contentés de pointer l'angélisme des approches normatives sans proposer d'alternative sur le plan pédagogique. Allons un pas plus loin : la nouvelle dialectique ne s'est pas véritablement vue opposer de nouvelle rhétorique. Pourtant, il y a bien une spécificité pédagogique de l'enseignement rhétorique. La rhétorique, depuis l'origine, vise à rendre les apprentis rhétoriciens plus lucides sur les interactions argumentatives. La rhétorique est, selon la définition d'Aristote, une faculté (dunamis) à porter un regard théorique (theoresai) sur les moyens de persuasion. C'est l'activation, par l'exercice, de ce regard théorique, qui est la condition nécessaire à l'acquisition de toute une gamme de compétences civiques dont nous avons plus haut commencé à effleurer la liste. Nous soutenons que l'activation de ce regard théorique suppose un enseignement rhétorique par l'exercice. En appui de cette thèse, nous avons construit une preuve que nous espérons, à défaut d'être irréfutable, du moins élégante. C'est cette preuve que nous proposons de livrer en ouverture de ce dossier.

\section{Le regard rhétorique}

3 Ainsi, nous avons voulu tester la capacité de publics contemporains à porter un regard théorique sur l'argumentation. Le rhétoricien est, en effet, celui qui s'est habitué à comparer les qualités respectives de différentes stratégies rhétoriques dans une situation argumentative donnée. Notre test visait à vérifier l'état de développement de cette compétence chez un public sans formation rhétorique préalable. Pour ce faire, nous avons demandé à des élèves du secondaire et à des étudiants de niveau universitaire de reconnaître les stratégies et les ressources mises en œuvre dans une "controverse", un exercice qui se présente comme une simulation simplifiée de procès ${ }^{3}$. La comparaison des performances des élèves et étudiants était pour nous un moyen de vérifier si le développement de la compétence rhétorique supposait bel et bien une formation rhétorique ou si elle se développait naturellement avec la maturation des facultés cognitives. Le test se composait de deux controverses et de six arguments orientés vers des conclusions opposées; en outre, ceux-ci étaient formulés dans un langage contemporain et quotidien, avec parfois une touche d'amplification, tels qu'ils pourraient apparaître dans les contextes envisagés. L'énoncé demandait 
d'apparier les arguments qui se ressemblaient et de fournir une brève justification ; un exemple était également fourni. 
Le test de rhétorique

$\left.1^{\circ}\right)$ Lisez attentivement les deux controverses suivantes et les arguments fournis

\section{Controverse $1:$ : Balle perdue»}

Énoncé

Il est environ 10 h30 lorsque que le professeur d'histoire vient surveiller la cour de récréation. Les élèves jouent un match de basketball et la partie semble très animée. Alors qu'il a le dos tourné, le professeur reçoit le ballon en pleine tête et est légèrement blessé. Il identifie sans peine l'auteur du tir, un élève turbulent qu'il a dû sanctionner à plusieurs reprises pour ses comportements inappropriés. Il l'accuse cette fois d'avoir commis une agression et demande son renvoi.

Arguments

Pour

1) «Si nous ne punissons pas cet élève, nous donnerons un mauvais exemple aux autres. »

2) «Si un État envoyait une bombe par accident dans un pays voisin, il s'exposerait à des représailles ou des sanctions internationales. »

3) «Si le ballon a pu blesser le professeur, il peut être considéré comme une arme et le tir comme une agression.»

4) «De toute manière, c'est le pire élève qu'on ait jamais vu : il est déjà en échec dans toutes les matières et est à peine capable de lire et d'écrire. Il vaut mieux qu'il quitte l'école. »

5) «Il a frappé à un endroit vulnérable et dans le dos, quand le professeur ne pouvait pas voir le coup arriver. C'est non seulement grave, mais en plus, c'est lâche."

6) «L'occasion de se venger des sanctions subies était trop belle et la précision du tir ne laisse aucun doute sur les intentions de l'élève. C'est un acte délibéré qui mérite d'être durement sanctionné. »

7) «Rares sont les professeurs qui ont encore la vocation, car c'est un métier difficile, mal payé et souvent ingrat; toute l'année, il faut composer avec les inspecteurs, les réformes, les parents mécontents, les élèves turbulents. Faut-il encore qu'ils subissent les insultes ou les tirs de ballon sans broncher? Qui pense à eux? »

\section{Controverse $2:$ «La part de gâteau »}

\section{Énoncé}

Leila est allergique aux noisettes. Elle a été élue présidente de la classe et est chargée de veiller au bon déroulement des cours. Elle adresse régulièrement des avertissements à ceux qui perturbent les leçons, et en particulier à Matthieu, qu'elle a un jour dû faire sortir de la classe. Toutefois, en l'honneur de son anniversaire, Matthieu lui offre une part de gâteau au "Nutella ( qui contient des noisettes). Leila le mange et est très vite prise d'un malaise. Elle est emmenée d'urgence à l'hôpital. Matthieu est accusé d'avoir empoisonné Leila et est menacé de renvoi.

Arguments

Contre

a) «J'ignorais qu'offrir une part de gâteau puisse être un crime. Aucune loi ou règlement ne l'interdit. Faut-il désormais mettre les pâtissiers sous surveillance? Ce serait le comble du ridicule. »

b) «Condamne-t-on un vendeur d'armes car un acheteur s'est suicidé avec l'arme qu'il lui a vendue? »

c) « On va encore punir ce pauvre Matthieu alors qu'il voulait simplement être gentil et tentait de se racheter. C'est injuste!»

d) «Leila a été élue parce qu'elle est populaire. Elle est prête à tout pour se faire remarquer et se hisser audessus des autres. Elle s'est arrangée pour se poser en victime et attirer la pitié sur elle. »

e) "Si Matthieu est renvoyé maintenant et pour ce motif, il ne trouvera jamais d'autre école et n'aura rien appris, à part le dégoût de l'école."

f) «Matthieu fêtait son anniversaire : c'est un moment de joie et de fête où l'usage veut que l'on s'amuse et l'on partage un gâteau. On ne peut pas reprocher à Matthieu d'aimer le Nutella et d'avoir fait preuve de savoir-vivre. »

g) «Matthieu ne savait peut-être pas que le Nutella contenait des noisettes ou a simplement oublié que Leila était allergique. Ce n'est qu'un regrettable accident. »

$\left.2^{\circ}\right)$ À présent, comparez les arguments avancés pour chacune des deux controverses. Dans le

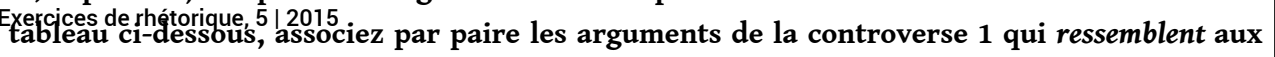
arguments de la controverse 2 . Pour chaque association, justifier votre choix, comme dans l'exemple. 


\begin{tabular}{|c|l|l|}
\hline $\begin{array}{l}\text { Arguments } \\
\text { controverse 1 }\end{array}$ & $\begin{array}{l}\text { Arguments } \\
\text { controverse 2 }\end{array}$ & \multicolumn{1}{|c|}{ Justification } \\
\hline $\mathbf{1}$ & e & $\begin{array}{l}\text { car ces deux arguments sont tirés des } \\
\text { conséquences de la décision }\end{array}$ \\
\hline & & \\
\hline & & \\
\hline & & \\
\hline & & \\
\hline & & \\
\hline & & \\
\hline
\end{tabular}

$\square$

\section{Plus que de l'argumentation : de la rhétorique}

4 Notre test de rhétorique s'inscrit dans une démarche d'affirmation disciplinaire. Depuis 2013, nous sommes en effet engagés dans un programme de recherche visant à réintroduire une formation rhétorique dans l'enseignement secondaire et universitaire ${ }^{4}$. Au plan théorique, un enjeu important pour nous est de réfuter l'idée selon laquelle la rhétorique ne serait qu'un vernis persuasif dont on pourrait enduire la logique et l'argumentation. Cette idée a pour corollaire une conception de l'éducation selon laquelle il faudrait, d'abord et avant tout, enseigner l'argumentation. Dans cette perspective, la rhétorique serait une discipline sophistiquée et en rien une priorité éducative. À l'appui de cette idée, les tenants des approches normatives ont, dès le milieu des années 1990, élaboré des tests d'argumentation ${ }^{5}$. Ces tests visaient à évaluer la capacité des participants à identifier les prémisses implicites d'arguments donnés. En effet, dans la perspective des approches normatives de l'argumentation, le travail critique commence par une activité de reconstruction. Il s'agit de reformuler les arguments effectivement produits pour les faire correspondre à des formes canoniques. Ces formes canoniques sont systématiquement associées à des questions critiques et distinguées de formes fallacieuses. Par exemple, une fois qu'un étudiant aura identifié un argument comme causal, il pourra tester la solidité du lien de cause à effet. Il pourra, en outre, vérifier que cet argument causal se distingue bel et bien de formes fallacieuses, comme l'argument de la pente glissante ou le sophisme du post hoc, ergo propter hoc (présenter comme une relation causale ce qui n'est qu'une succession entre deux événements). Le test mis en œuvre par les tenants de l'approche normative révèle des difficultés chez les participants dans l'identification des prémisses implicites. Il offre donc une justification à l'introduction d'une formation à l'argumentation dans les programmes scolaires.

5 L'épistémologie et le programme pédagogique des approches normatives de l'argumentation sont cohérents et convaincants, sur des plans autant éthiques que 
politiques. En effet, on peut légitimement penser que plus les citoyens seront armés pour soumettre les arguments à un examen critique rigoureux, plus ils pourront être exigeants à l'égard de leurs interlocuteurs et plus le niveau global du débat s'élèvera. Pourtant, notre test de rhétorique nous permet de soutenir une perspective différente, voire opposée. Notre test révèle en effet que les quelques notions d'argumentations dont disposent les participants sont précisément celles que proposent d'enseigner les approches normatives de l'argumentation: il ne faut pas attaquer sur l'ethos, le pathos est suspect et on n'argumente pas en dehors de la cause ${ }^{6}$. Allons un pas plus loin dans l'interprétation : notre test révèle que le regard des participants est confisqué par une épistémologie spontanément normative, ce qui empêche toute finesse dans l'analyse. Deux arguments seront, généralement, considérés comme de même nature au regard du simple fait qu'ils seront perçus comme fallacieux. Or, porter un regard technique sur l'argumentation, comme nous le proposons, requiert au contraire de reconnaître les éléments de la technique, discerner les stratégies, preuves et lieux exploités parmi ceux qui sont disponibles, mettre un nom sur les procédés abstraits observés à travers leur réalisation concrète. Il s'agit d'apprécier les qualités (et, par effet de miroir, les défauts ou les faiblesses) d'une argumentation ou, pour une reprendre une analogie avec la médecine clinique ${ }^{7}$, d'identifier les symptômes, qui s'expriment de manière plus ou moins évidente et aiguë, et conjointement ou non à d'autres.

\section{La critique rhétorique : une conscience des alternatives}

6 Avant de passer à l'analyse des résultats, il convient d'apporter une précision sur le rôle $\mathrm{du}$ professeur de rhétorique en comparaison du professeur de logique et argumentation. Le professeur de logique et argumentation énonce les règles et sanctionne les erreurs. Le professeur de rhétorique propose des exemples et se donne en exemple. En effet, en rhétorique, la critique est une activité plus comparative que normative. Le travail du professeur consiste à montrer la multiplicité des stratégies disponibles pour la défense d'une opinion. C'est la conscience de cette richesse qui formera des citoyens prêts à argumenter dans les situations argumentatives les plus diverses. Il en résulte que la correction d'un exercice de rhétorique est une proposition. S'il est attendu que le professeur fasse mieux que ses élèves dans un exercice donné, les performances des étudiants doivent être analysées comme des alternatives rhétoriques possibles et non comme des déviances par rapport à un modèle. Voici donc une proposition de corrigé du test de rhétorique, fondée sur une connaissance technique des lieux et des preuves 8 .

\begin{tabular}{|l|l|l|}
\hline 1 & e & Les deux arguments sont tirés des conséquences probables de l'acte envisagé (lat. ab effectis) \\
\hline 2 & b & Les deux arguments s'appuient sur des analogies (lat. a simili, a similitudine) \\
\hline 3 & a & $\begin{array}{l}\text { Les deux arguments reposent sur la définition (lat. finitio), qu'il s'agisse de la restreindre ou } \\
\text { de l'élargir (lat. syllogismus) }\end{array}$ \\
\hline 4 & d & $\begin{array}{l}\text { Les deux arguments sont tirés de la personne de l'accusé ou de la victime (a persona; } \\
\text { ad hominem) }\end{array}$ \\
\hline
\end{tabular}




\begin{tabular}{|l|l|l|}
\hline 5 & $\mathrm{f}$ & On tire argument des circonstances (lat. ab adiunctis) \\
\hline 6 & $\mathrm{~g}$ & $\begin{array}{l}\text { On discute de l'intention réelle ou supposée de l'accusé ou de la victime (lat. a causa, } \\
\text { ab intentione) }\end{array}$ \\
\hline 7 & $\mathrm{c}$ & $\begin{array}{l}\text { Les deux arguments, s'ils peuvent être compris de différentes façons (intention, contraires, } \\
\text { conséquences), font un appel franc à l'émotion (pitié, indignation) }\end{array}$ \\
\hline
\end{tabular}

Cette lecture a guidé l'élaboration du test: nous voulions savoir si les personnes interrogées étaient capables de ressentir des similitudes et des différences au niveau du logos et de les exprimer, de détecter des émotions, de passer au-delà des procédés de style et d'amplification pour proposer un ensemble d'associations cohérentes. Une nouvelle fois, il faut se garder de voir cette analyse comme une grille normative qui permettrait de trancher chaque cas et considérer ces associations comme les seules bonnes réponses possibles et qui permettraient, sans autre procès, de sanctionner les mauvaises. Si l'on est parfois tenté d'appliquer les principes rhétoriques comme ceux d'une grammaire, la réalité se laisse malaisément enfermer dans les concepts théoriques utilisés pour la décrire ; aucun discours, si bref soit-il, aucun argument en contexte, s'il est réel ou réaliste, ne recouvre exactement le type ${ }^{9}$, sorte d'idéal, auquel il est censé appartenir, car il n'y a d'argument que relativement à un contexte particulier. Dans la pratique, on peut très bien sembler vouloir exploiter plusieurs ressources à la fois : les trois preuves techniques définies par Aristote sont des outils commodes, mais ne fonctionnent pas comme des catégories étanches. De même, au niveau du logos, il n'est pas faux de dire que l'argument (f) s'appuie également sur l'intention, ou que l'argument (5), qui qualifie l'action de «lâche ", tend également à disqualifier la personne; on peut toujours hésiter entre des distinctions subtiles, qui en contexte, se confondent volontiers. Pour trancher, on peut invoquer un principe de dominance (dans 5-f, le "focus" est mis sur les circonstances et la manière dont l'acte a été accompli ; dans 7-c, sur l'émotion), mais même un tel critère laisse inévitablement une zone de flou et une place pour de multiples interprétations, qu'on ne peut qu'accepter; dès lors, l'appréciation technique reste ouverte à la plurivocité, à l'enrichissement ou à la critique et la remise en question: on pourrait très bien invoquer d'autres critères (les émotions, les traits de caractères, le point visé) ou exploiter d'autres typologies cohérentes ${ }^{10}$. Les rhéteurs, anciens ou modernes, n'étaient pas toujours d'accord entre eux sur la manière de traiter tel sujet, sur la compréhension de tel discours, l'évaluation de tel argument et sur la façon de le nommer; leur lecture n'en demeurait pas moins faite à partir de la technique, qui affûtait leur regard. Les éléments de la technique, dont on trouve le détail dans les traités anciens, sont des outils conçus pour décrire et comprendre la pratique, qui ellemême permettait de les questionner sans cesse et pouvait être guidée par ceux-ci, sans pour autant y être absolument assujettie, comme à des normes; une tension, ou tentation, toujours présente. En associant des paires d'arguments selon les critères dont ils disposaient, les personnes que nous avons interrogées ont accompli la tâche qui leur était demandée : il ne s'agit pas pour nous de savoir si leurs réponses sont vraies ou fausses, mais bien d'observer la manière dont ils pensent face aux arguments qu'ils ont sous les yeux. 


\section{Analyse des résultats}

En comparaison des élèves, les étudiants ont généralement eu des intuitions sur les ressemblances entre les arguments plus en accord avec les couples que nous avions à l'esprit en préparant le test. Cela pourrait signaler une compétence à l'abstraction plus mature que celle de leurs benjamins. Les données qualitatives récoltées (la manière dont les participants justifient leurs associations) légitiment l'objectif de la formation rhétorique. En effet, si les étudiants ont la capacité de porter un regard théorique sur l'argumentation, celle-ci doit encore être sculptée.

\subsection{Le regard rhétorique des élèves}

Deux classes de 20 et 23 élèves de deuxième année secondaire ${ }^{11}$ ont été soumis au test pendant 45 minutes, avec les résultats suivants ${ }^{12}$.

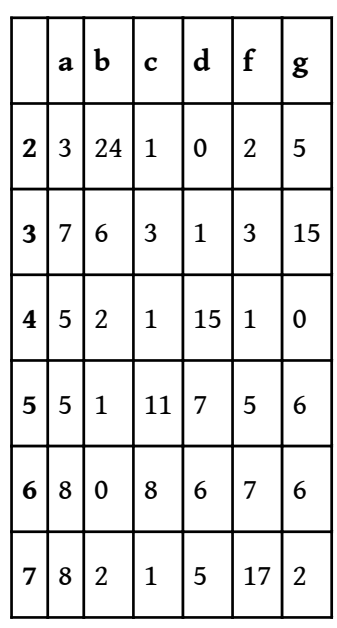

10

On constate tout d'abord l'éclatement des réponses, malgré quelques associations privilégiées: le couple 2-b a largement été identifié comme un exemple ou une comparaison, le couple 4-d autour de la personne; les élèves ont également été sensibles aux tentatives de susciter la pitié, terme qui revient à plusieurs reprises dans leurs justifications. Au niveau de ces dernières, les élèves éprouvent parfois des difficultés à exprimer les similitudes qu'ils repèrent, mais de manière générale, ils procèdent par libres associations d'idées, exploitent les quelques notions abstraites qu'ils manipulent ("vengeance", " injustice», " pitié », " exemple», « contraire», " intention ", " mobile ») ou restent très proches du texte (" ces arguments parlent ", " on parle de l'élève ", « des faits ", « de l'arme ", « du crime ", « de la victime ", « de son caractère ") avec une répartition qui s'organise autour de l'opposition persona et res, généralement sans a priori négatif (le commentaire "mal argumenté » n'apparaît qu'une seule fois). Des notions floues comme l'intention, la manière ou les émotions ressenties à la lecture des arguments semblent être à l'origine de l'éclatement des réponses dans certains cas. Si donc l'esprit des élèves n'est pas vierge de tout concept ayant un lien avec la rhétorique, il reste à construire et peut être ouvert à 
l'introduction de nouvelles notions comme à la modification et la réorganisation de celles qui sont présentes.

\subsection{Le regard rhétorique des étudiants}

11 Nous avons soumis le même test à 50 étudiants de l'Université Libre de Bruxelles, avec les résultats suivants ${ }^{13}$ :

\begin{tabular}{|l|l|l|l|l|l|l|}
\hline & $\mathbf{a}$ & $\mathbf{b}$ & $\mathbf{c}$ & $\mathbf{d}$ & $\mathbf{f}$ & $\mathbf{g}$ \\
\hline $\mathbf{2}$ & 6 & 39 & 0 & 0 & 1 & 4 \\
\hline $\mathbf{3}$ & 27 & 8 & 1 & 1 & 5 & 5 \\
\hline $\mathbf{4}$ & 1 & 0 & 9 & 35 & 4 & 0 \\
\hline 5 & 2 & 1 & 15 & 1 & 15 & 12 \\
\hline 6 & 2 & 0 & 3 & 7 & 9 & 24 \\
\hline 7 & 8 & 2 & 18 & 7 & 11 & 3 \\
\hline
\end{tabular}

En comparaison des élèves du secondaire, les étudiants ont la capacité à porter un regard théorique sur l'argumentation: leurs intuitions sur la ressemblance entre les arguments sont plus solides que celles de leurs jeunes camarades. La dispersion des réponses pour les arguments 5,7 , c et $f$ s'explique, comme nous l'avons $v u$, par l'ambiguïté de la stratégie rhétorique. L'enseignement le plus intéressant du test ressort de la prise en compte des données qualitatives.

Les commentaires des étudiants sur les critères de leurs choix de coupler deux arguments témoignent des enjeux qu'il y a à éduquer le regard théorique par l'exercice rhétorique. Le problème principal que nous avons identifié est le normativisme de l'épistémologie spontanée des participants. Ce normativisme les empêche de percevoir la diversité des stratégies rhétoriques ${ }^{14}$. Prenons l'exemple d'un étudiant qui rapproche les arguments (2) et (b) en ce qu'ils sont des « arguments amenés dans une proportion beaucoup plus grande, exagérés ». On peut envisager qu'un tel jugement normatif l'empêchera de percevoir l'argument par analogie comme une technique pertinente et réutilisable. En outre, il est intéressant de noter que beaucoup d'élèves se sont placés dans une position de vigilance à l'égard des arguments fallacieux, posture qui n'était pas induite par l'exercice (il s'agissait uniquement de justifier une ressemblance entre les arguments). Enfin, certains étudiants semblaient agacés par les arguments observés. Il semble donc que, spontanément, nous soyons disposés à sanctionner les arguments qui s'écartent de nos idéaux normatifs. Par exemple, en justification du couple 3-a, un étudiant écrivait : "arguments qui amènent une interprétation abusive, trop facile ${ }^{15}$. " De ces données qualitatives, nous proposons l'interprétation suivante : il semble que l'épistémologie spontanée des étudiants appauvrisse leur regard théorique sur l'argumentation. Ce normativisme pourrait bloquer la capacité à observer la diversité des stratégies argumentatives. Le travail du professeur de rhétorique consistera alors à 
enrichir, à densifier le regard de ses étudiants sur l'argumentation. Les contributions à ce dossier s'inscrivent dans cette perspective.

\section{Présentation du dossier}

Benoît Sans ouvre le dossier par un travail de rétro-ingénierie : retrouver les secrets de fabrication d'exercices de rhétorique antiques à partir de leurs énoncés. Ces exercices, à l'instar de la controverse qui est l'objet d'étude de l'auteur, nous sont parvenus sans les plans. Pour cause, l'objectif des rhéteurs était de former des orateurs et non des professeurs. Or l'élaboration d'une controverse suppose un subtil dosage de faits et de zones d'ombres, de liberté et de contraintes, permettant de canaliser l'imagination de l'élève au profit de sa créativité. Grâce à la contribution de Benoît Sans, les professeurs pourront se réapproprier l'exercice de base de l'orateur et transmettre à leurs élèves le plaisir intellectuel de l'inventio.

Roberta Martina Zagarella et Marco Mazzeo proposent ensuite d'apprivoiser deux stratégiques rhétoriques : la résistance à la calomnie et l'attaque verbale. L'analyse de Roberta Martina Zagarella porte sur le film Changeling de Clint Eastwood et, plus précisément, sur le combat de son personnage principal contre le mensonge et la manipulation. Comment défendre la vérité à la face du monde ? Comment ne pas devenir fou à force d'être perçu comme tel ? Résister à la calomnie demande de solides ressources éthiques, que le lecteur découvrira décrites avec brio.

L'article de Marco Mazzeo met en lumière un objet rhétorique exceptionnel : l'attaque verbale chez Muhammad Ali. L'agression par les mots, si présente au quotidien, est marginale au plan théorique. Les analyses de Marco Mazzeo en livrent la raison: les mots qui agressent se situent à la frontière entre logos et praxis. De ce fait, ils passent généralement sous le radar de l'œil théorique du rhétoricien. En livrant à son lecteur les secrets du boxeur-orateur, Marco Mazzeo le prépare, du même coup, à la contreattaque et à l'esquive.

Le dossier se poursuit par une proposition, formulée par Emmanuelle Danblon et Ingrid Mayeur, de reconnaître un nouveau genre rhétorique, apparenté mais distinct des genres délibératif et épidictique : un genre utopique. Pour les deux auteurs, cette fonction utopique se manifesterait tout particulièrement dans les chartes et déclarations. En l'occurrence, leur analyse porte sur un objet rhétorique en construction : la Déclaration préliminaire des Droits de l'Homme Numérique. Le lecteur aura compris que la nouveauté ne réside pas dans le genre en lui-même : il y a bien longtemps que l'utopie habite l'imaginaire des sociétés humaines. Ce qui est inédit, en revanche, est l'appropriation de cette fonction utopique par la technique rhétorique. Désormais, nous aurons entre les mains les outils pour exercer l'utopie.

Pour finir, Victor Ferry propose une approche rhétorique de l'empathie. L'empathie, bien que jamais nommée dans les traités, est une compétence au cœur de la tradition rhétorique. Qu'est-ce, en effet, qu'un orateur, sinon un expert de l'empathie? L'adaptation des arguments à l'auditoire suppose une simulation mentale de la subjectivité du public. Victor Ferry étudie l'usage rhétorique de l'empathie chez Barack Obama, dans son discours du Caire, par lequel il entendait impulser un nouveau départ dans les relations entre les États-Unis et le monde musulman. L'analyse révèle une forme d'excès de confiance, chez l'orateur, dans sa capacité à dépasser les divergences d'opinions sur les différents sujets abordés. Partant, l'auteur montre, qu'à l'état brut, 
notre compétence à l'empathie est souvent mauvaise conseillère. Éduquer notre empathie passe par la maîtrise de compétences connexes parmi lesquelles la prudence et le tact occupent une place de choix. Le lecteur trouvera ici les ressources théoriques et les conseils pratiques pour faire ses gammes dans les rhétoriques de l'empathie.

\section{NOTES}

1. M. Angenot, Dialogue de sourds, Paris, Fayard, 2008.

2. Voir sur ce point J.-L. Dessalles, La pertinence et ses origines cognitives, Paris, Hermès, 2008.

3. Pour plus de détails sur ce type d'exercice, sur la manière dont les controverses étaient pensées et pourraient être adaptées, voir l'article de Benoît Sans dans le présent numéro.

4. La tradition nous a livré quantité d'exercices variés, longtemps délaissés et que nous essayons de remettre au goût du jour. Outre les traités théoriques (Aristote, Cicéron, Quintilien...), nous avons conservé des manuels d'exercices (Aélius Théon, Ps.-Hermogène, Aphtonios, Nicolaos) et des recueils de déclamations (Sénèque le Rhéteur, Ps.-Quintilien, Sopatros) auxquelles s'ajoutent de nombreuses indications ou énoncés figurant dans les traités de rhétorique, les ouvrages de sophistes et rhéteurs (Aelius Aristide, Lucien), dans leur biographie (Suétone, Philostrate) ou leur correspondance (Libanios, Fronton), ainsi qu'une documentation papyrologique grandissante. Victor Ferry et Benoît Sans testent actuellement les exercices de rhétorique, repris ou inspirés de ceux qui étaient pratiqués dans l'Antiquité, au niveau secondaire et universitaire dans le cadre d'un projet de recherche intitulé «Exercices de rhétorique: raison pratique, créativité, citoyenneté ", dirigé par Emmanuelle Danblon et financé par le Fonds National Belge de la Recherche Scientifique (FNRS). Pour plus de détails, voir l'article de B. Sans dans le présent numéro. Les ressources pédagogiques créées dans le cadre de ce projet sont disponibles sur le site du Groupe de recherche en Rhétorique et en Argumentation Linguistique (GRAL): http:// gral.ulb.ac.be/democracy-lab.

5. F. H. V. Eemeren, K. D. Glopper, R. Grootendorst et R. Oostdam, «Identification of unexpressed premises and argumentation schemes by students in secondary school ", Argumentation and Advocacy, 31 (3), 1-15, 1995.

6. Les tenants de l'approche pragma-dialectique de l'argumentation devraient d'ailleurs nous rejoindre sur ce point. Dans un article publié en 2012, Frans van Eemeren, Bart Garssen et Bert Meuffels montraient empiriquement que les normes utilisées par les locuteurs ordinaires dans leurs évaluations des arguments étaient en accord avec celles enseignées par la théorie pragmadialectique de l'argumentation. Voir : F. van Eemeren, B. Garssen et B. Meuffels, « The extended pragma-dialectical argumentation theory empirically interpreted» dans Topical Themes in Argumentation Theory, Springer Netherlands, 2012, p. 323-343.

7. Analogie empruntée à Fr. Goyet, "Le problème de la typologie des discours ", Exercices de rhétorique [En ligne], 1|2013, mis en ligne le 02 juillet 2013, consulté le 15 mars 2015. URL: http://rhetorique.revues.org/122 ; DOI : 10.4000/rhetorique.122.

8. Nous avons choisi ici l'approche et les dénominations latines qui remontent à la Rhétorique à Herennius, Cicéron et Quintilien, mais qui sont également exploitées par les maîtres de rhétorique de la Renaissance ou de l'Époque Classique (on consultera avec profit le lexique fourni par l'équipe «Rhétorique de l'Antiquité à la Renaissance » [RARE] sur son site internet). Pour les 
termes grecs, voir notamment les lexiques de L. Pernot, La rhétorique dans l'Antiquité, Paris, Librairie générale française, 2000 et D. A. Russell, Greek Declamation, Cambridge, Cambridge University Press, 1983 [2009].

9. Ce principe est également applicable au niveau des discours eux-mêmes (Fr. Goyet, op. cit.)

10. Comme celle qui a été élaborée par Chaïm Perelman et Lucie Olbrechts-Tyteca dans le Traité de l'argumentation.

11. L'équivalent de la classe de cinquième dans le système français.

12. Il est à noter qu'un élève n'a pas répondu au test et que plusieurs d'entre eux n'ont répondu que partiellement.

13. Là encore, à noter que certains étudiants ont rendu des questionnaires partiels.

14. Il ne s'agit pas du seul problème que l'analyse des données qualitatives permet de révéler. Un autre problème est celui de l'imprécision des catégories utilisées pour classer les arguments. Par exemple, lorsqu'un étudiant associe l'argument (3) à l'argument (a) en ce qu'ils sont « des appels à la raison ", la catégorie " appel à la raison » est trop vague et trop imprécise pour qu'on puisse en tirer un moule pour produire des arguments dans un autre contexte.

15. Victor Ferry a proposé le concept d'illusion normative pour interpréter ce phénomène. L'illusion normative se déclenche lorsque nous percevons la dimension technique de l'argumentation. Cette perception, pour qui n'est pas acquis à la cause de la rhétorique, entraîne la suspicion : l'artifice sera systématiquement interprété comme l'indice d'une erreur ou d'une tromperie. Ainsi, l'idéal normatif inhibe la compétence critique en faisant de la perception du caractère artisanal des preuves un motif de blâme. Dans une perspective rhétorique, au contraire, c'est la perception du caractère artificiel des preuves qui est le point de départ du travail technique en vue de les perfectionner. Sur ceci, voir V. Ferry, La raison humaniste, Paris, Classiques Garnier, à paraître.

\section{AUTEURS}

\section{VICTOR FERRY}

University of Oxford - GRAL (Groupe de recherche en Rhétorique et en Argumentation Linguistique)

\section{BENOÎT SANS}

Université Libre de Bruxelles - GRAL (Groupe de recherche en Rhétorique et en Argumentation Linguistique) 